\title{
Research on the Application of Knowledge Discovery in Digital Library Service
}

\author{
Huanyu Zhu ${ }^{1, \text { a }}$ and Xin $\mathrm{Li}^{1, \mathrm{~b}^{*}}$ \\ ${ }^{1}$ Library, Beihua University, Jilin, China \\ ayurix999@sina.com.cn, b35780861@qq.com \\ ${ }^{*}$ Corresponding author
}

Keywords: Knowledge discovery; Digital library service; Application

\begin{abstract}
This paper explores the function and general process of knowledge discovery by exploring the knowledge discovery and the general process. Knowledge delivery services and reference services.
\end{abstract}

\section{Introduction}

With the rapid development of network environment and database technology, human had stepped into the Digital information age, people's understanding of the world more and more comprehensive and in-depth. Correspondingly, the various data information grow exponentially and cover all aspects of our lives. In these vast numbers of data, hidden a lot of important information. Although the current database technology can efficiently and easily achieve the query function of data, but according to the existing data retrieval mechanism and statistical analysis method still can not meet some high-level needs, can not proceed from the user's needs, to achieve user access Accurate information on the intention of personalized active knowledge services even more out of the question." Although the data is rich, but the information is still scarce," users are still in the information overload environment facing a difficult choice. Digital library as a collection of data ,information, knowledge as a treasure trove of knowledge, but also faced with a series of problems described above, how to apply knowledge discovery technology to the construction of digital libraries to comprehensively enhance the value of digital library knowledge services And innovation is a new era, the new situation to bring the challenges of the library.

\section{Knowledge Discovery}

The Concept of Knowledge Discovery. The term knowledge was first proposed at the International Joint Artificial Intelligence Conference held in 1989. There are several versions of the definition, which is currently widely recognized by Fayez. Knowledge discovery refers to the high-level process of obtaining effective, unknown, potentially valuable and ultimately understandable patterns from large amounts of data. Knowledge discovery is a cross-disciplinary discipline developed in the field of computer science, database technology, machine learning techniques, artificial intelligence, statistical techniques and other disciplines. Knowledge discovery is essentially a systematic process, the core of which is the implementation of a large number of data warehouses, knowledge base, database analysis and in-depth mining, find the data between the potential correlation, rules, patterns, trends. To help users find the data behind the hidden knowledge and information, compared with the traditional information retrieval, knowledge discovery to obtain the information is the previous unknown, effectiveness and practicality of the three characteristics.

Function of Knowledge Discovery. Knowledge discovery is different from the traditional database orientation query, retrieval, call, it will analyze the data, statistics, classification and reasoning, as a basis to guide the practical problem solving, and trying to find the interconnection between the affairs, according to this Data interlinkages predict future events. Knowledge 
discovery technology can be from the massive, messy data according to the specific algorithm, screening out the valuable, high-quality associated data.

The General Process of Knowledge Discovery. On the process of knowledge discovery, there are still differences, some scholars put forward "five steps to say", there are scholars put forward "six step", this paper that knowledge discovery process should be divided into nine steps. First step, user survey, to understand the needs of users. Second step, data collection, the creation of the target data set, the data set can be from the existing system, but also from the data warehouse. Third step, data preprocessing and Clean up, correct the previous database and remove the redundant data, and convert the results into a standard form of data acquisition tools. Forth step, data simplification and projection, to find out the effective characteristics of data mining objectives, reduced dimension processing or equivalent with other variables to reduce the effective number of variables. Fifth step, choose the appropriate data mining method according to the target. Sixth step, Select the appropriate data mining algorithm according to the mode type. Seventh step, using the selected data mining methods and algorithms for data mining, mining users interested in the model. Eighth step, Evaluate and explain the patterns found and visualize them. Ninth step, knowledge collation and application, the results of the excavated knowledge are applied to the user's system. The above steps can be further summarized into three parts: data preparation phase, data mining stage and data mining post-processing stage. The model is shown in Figure 1.

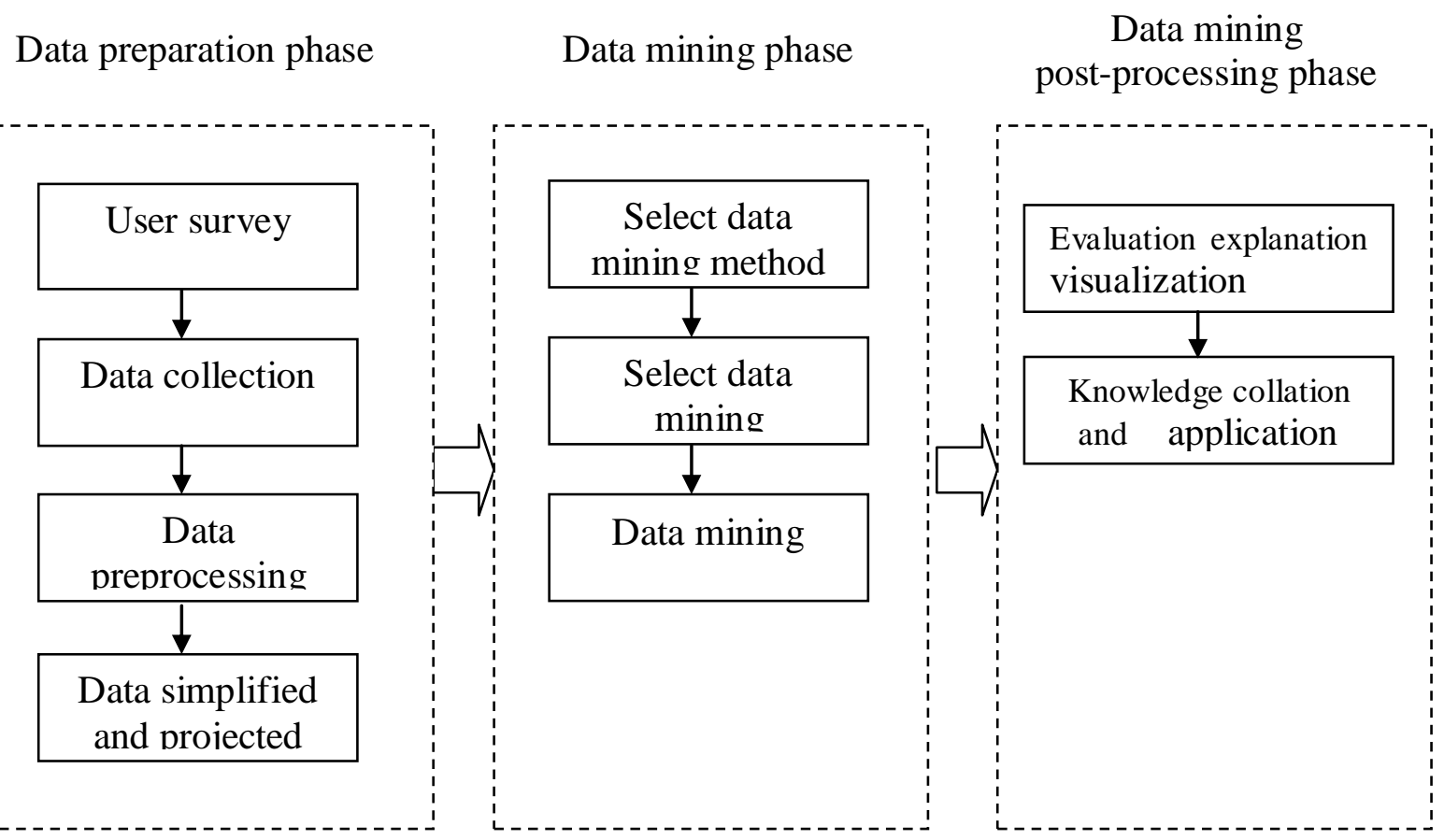

Figure 1. The general process of knowledge discovery

\section{The Significance of Introducing Knowledge Discovery System into Digital Library}

To Improve the Utilization Rate of Digital Library Resources Helpfully. In the traditional digital library resources construction, simply pay attention to the resource construction itself and the retrieval transmission ability, while ignoring the user for the use of resources efficiency problems, resulting in the digital library resource utilization is generally low status quo. The introduction of knowledge discovery system makes the digital library on the basis of the original resources, through the correlation analysis, classification, clustering and other methods, access to the original information there is a certain correlation and hidden in the literature, data, the valuable knowledge implied under the data. The different from the traditional model, knowledge discovery system for user access to knowledge A at the same time, will also have a certain relevance of knowledge B. Provided to the user for selection. This push not only increases the utilization of knowledge B, but also allows users to obtain the knowledge which they can not get. From this we can see that the core 
of knowledge discovery is the mining of knowledge, it is exist for the needs of users, in the knowledge service model, should be knowledge to find users, not just limited to the user to seek knowledge. The application of knowledge discovery system effectively improves the utilization rate of digital library resources.

To Provide Users with Personalized Knowledge Services. Since entering the new century, data, information, knowledge has become more and faster. According to statistics, the world generated about $2 \mathrm{~EB}$ data every day (2EB both 1 billion GB or $1000 \mathrm{~PB}$ ), combined with the previous accumulated data, simply countless. In such a large amount of data is filled with a considerable part of the garbage data (including false data and redundant data), these data not only can not meet the needs of users, but also cause trouble. Knowledge discovery system through the collection, association analysis, clustering, classification and other methods, the site structure, resource content and user situation in-depth mining to find out the information characteristics and information associated with and make reasonable use, improve the level of knowledge services, and thus promote the user's Information literacy. In addition, the digital library knowledge discovery system, by tracking and analyzing the characteristics of user information and its knowledge and behavior, uses the method of association analysis, classification and time series analysis to excavate the regularity and characteristics of its existence as a digital library resource intelligent association And clustering basis. Through the knowledge discovery technology on the available knowledge of the association and appreciation, and then use these data and laws of the user's future activities to make a reasonable prediction, so as to push the user knowledge to meet the user's knowledge and practical needs, to achieve from the information to the knowledge service across.

To further promote the development of digital library resources and database construction. The data resources is very valuable, through the knowledge discovery system to identify potential resources in the characteristics and laws to provide users with the needs of users, efficient access to knowledge, so that users can get the knowledge potential, newly, Valuable, to achieve the use of resources to maximize, to eliminate the unnecessary loss of knowledge resources, and as a guide to optimize the allocation of digital library resources. The knowledge discovery system strengthens the development of the existing digital resources and the potential link between the multi-dimensional resources, thus reducing the blind purchase of resources and reducing the inability of the existing resources to be fully utilized. Through the analysis of user information and behavior characteristics, understand the needs of users, and then guide the development of digital library resources and database construction, update and optimization.

\section{The Application of Knowledge Discovery in Digital Library Service}

Knowledge Retrieval Service Based on Knowledge Discovery. Knowledge retrieval is different from information retrieval, it is indexed at the semantic level, the establishment of knowledge base. Through the semantic concept of information, to reveal the inherent meaning of information, rather than literally pattern matching. Which solves the problem of serious redundancy, low retrieval rate and high knowledge cost. Digital library with knowledge discovery technology, analysis of user search behavior, mining their interested knowledge, build user information model. Clustering analysis of the user, usually can be divided into clear query, semi-fuzzy query, fuzzy query. Among them, the knowledge retrieval service can improve the query quality of the latter two queries. In the digital library retrieval system, knowledge discovery reveals the deep correlation of knowledge, which enhances its interaction, breaks the time and space constraints of knowledge dissemination and sharing, and makes one-stop knowledge retrieval service reality.

Personalized Knowledge Push Service Based on Knowledge Discovery. Through the use of records, user behavior mining, user characteristics classification and user feedback analysis, through the pattern recognition and machine learning, analysis and prediction what knowledge users interested in. According to the construction of the user interest model to push the relevant knowledge to the user, you can also cluster, analysis, association and other rules to find the same interest users to be classified, and the knowledge pushed to similar needs of the user. Knowledge discovery using intelligent analysis, digital library to the user's personalized push service and 
personalized customized services, and based on the user for the push or customized service feedback, and further adjust the strategy to meet the needs of users, improve the user experience.

Consultation services based on knowledge discovery. The reference service is the earliest to date back from the University of Maryland's "Reference Service Electronic Access" project. The traditional shallow reference service is unable to meet the needs of users, personalized knowledge value-added services in the digital library services in a dominant position. Knowledge consulting services based on the digital library of rich intellectual resources and information resources, relying on the knowledge discovery system to provide users with the original knowledge or deep processing of knowledge. Through the knowledge discovery system to the needs of users to locate, tap the potential needs of users in order to provide users with more accurate knowledge services. Knowledge-based reference services are not subject to time constraints, geographical restrictions, in a variety of forms, especially to provide users with efficient, convenient, deep-level knowledge services.

\section{Conclusions}

Knowledge discovery service is the future development direction of digital library knowledge service, advancing the accumulation, organization and collation of knowledge information, promoting the creation and sharing of new knowledge, and constantly enriching resources and services, which will become indispensable part in the digital library construction. The extensive application of knowledge discovery system in digital library will further enrich and optimize the information resources of the library, so that the library's knowledge service ability will change qualitatively.

\section{Acknowledgements}

This paper is the relevant research result of the Educational Science in Jilin Province " Research on the cultivation of College Students' search skills from the Internet Perspective"(project number: GH16050) ;This paper is the relevant research result of the Educational Science in Jilin Province " Research on the teaching model reform of Library Literature Retrieval Course under the prosperity of network culture"(project number: GH170053) .

\section{References}

[1] W.J Zhang: Library Theory and Practice, Library Theory and Practice, No.9,p. 83-85. (I $\mathrm{n}$ Chinese)

[2] Y.J. Li, X.X. Zhang and K.Y, Zhang: Library and Information Service,Vol:59(2015)No.6, p.90-96. (In Chinese)

[3] H.L. Shao: Library, No.2,p.70-73.(In Chinese)

[4] A.J. Xue and F. Li: Computer Systems \& Applications, Vol:25(2016)No.4,p.252-257. (In Chinese)

[5] S.Y. Zhang and P. Cui: Library Work and Study, No.4,p.58-61.(In Chinese) se)

[6] H.Y. Shi: Library Science Research, Vol:19(2010)No.10,p.36-39.(In Chinese)

[7] S.M. Guo and X. Liang: Journal of Academic Libraries, Vol:32(2014)No.1,p.90-96. (In C hine

[8] F.R. Li: Journal of Library Science, Vol:38(2016)No.8,p.108-111.(In Chinese)

[9] H.X. Fan: Modern Information, Vol:28(2008)No.8,p.90-92.(In Chinese)

[10] X. N. Jin and B.Y. Ding: Research on Library Science, No.9,p.48-51.(In Chinese)

[11] Z.J. Liao: Information Science, Vol:30(2012)No.12,p.1849-1853.(In Chinese)

[12] N. Wang:, Library Work and Study, No.4,p.58-61.(In Chinese) 